\title{
Prognostic Value of Serum Lactate Level in Umbilical Cord in Predicting Complications and Outcome of Meconium Aspiration Syndrome A.E.Elsadek ${ }^{1}$, O.M.Abdel Haie ${ }^{1}$, M.B.Hassanin ${ }^{2}$, A.A.El Fallah ${ }^{3}$ and M.I.El Bishbishy ${ }^{1}$ \\ ${ }^{1}$ Pediatrics, Dept., Faculty of Medicine, Benha Univ., Benha, Egypt \\ ${ }^{2}$ Fellow of pediatrics Galaa Teaching Hospital, Egypt \\ ${ }^{3}$ Clinical and Chemical Pathology, Dept., Faculty of Medicine, Benha Univ., Benha, Egypt \\ E-mail:mahyesmail@gmail.com
}

\begin{abstract}
Background: Meconium drawing is a life-threatening disorder. It is distinguished by significant respiratory difficulties. Lactate is a fast-paced variable, strongly related to difficulties and mortality in nearly every severely sick child. Our aim in this study was to anticipate difficulties with the drawing of meconium by lactate measurement. Methods: This is randomised control work, occurring in 40 babies with meconium stained liquid. From February 2020 until November 2020, they were allowed to attend Benha University Hospital. Results: The need for resuscitation of babies with meconium contaminated liquor was 60 percent. Administration of surfactants was 16 percent. Stay in the hospital, vary from (4-45) days. Diabetic mother's maternal history was $20 \%$ and hypertension maternity was $12 \%$. Infant issues report that $20 \%$ air leak syndrome, $16 \%$ air leak syndrome, $52 \%$ hypoxic ischemic encephalopathy and $64 \%$ required for breathing assistance. Air leak syndrome Conclusion: Lactate measurement may be utilised to prevent issues such air leak syndrome, ischemic encephalopathy and breathing assistance in babies receiving meconiumtreated liquor.
\end{abstract}

Keywords: Meconium Stained liquor, Outcome, Umbilical cord

\section{Introduction}

The drawing of meconium breath after delivery with meconium coloured body and liquor with or without air leakage characteristics is known to cause respiratory discomfort. Chest radiographs may reveal extensive, patchy or homogeneous opacity, infiltration and air trapping evidence. [1] Infants who take meconium from the breath more vulnerable to breathing, especially those without perinatal care. [2] Meconium-stained liquor is very troubling from both an obstetrician and a pediatrician's viewpoint, and it is necessary for foetal surveillance to minimise complications and mortality. [3]

When meconium is breathing, three processes impact the lung: airway blockage, inflammation and tensile malfunction. [4]

Lactate is generated with the greatest amount of production seen in muscle in most tissues in the human body. [5] The liver (20-30\%), renal cortex (20\%), and myocardial are the most significant lactateconsuming tissues (5-15 percent ). [6] In different situations the precise pathophysiology of high lactate is complex, patient-specific and disease-specific. In general, higher production, reduced clearance or combination of both may induce lactate rise. [7] Our objective in this study was to anticipate the difficulties of babies who use lactate to extract meconium from the breath.

\section{Patients and Methods}

It is randomized control work, that's occur on 40 infants who had liquor stained with meconium. They were gave permission to enter Benha University Hospital from February 2020 to January 2021.

\subsection{Inclusion criteria}

- Infants with liquor stained with meconium.

- Infants equal to or higher than 38 weeks.

2.2. Exclusion criteria

- Preterm or near term infants.

- Other cause of difficult of breathing.

2.3. Methods

A) Examination of infant including

1) Determination of sex: (male or female).

2)Assessment of vital signs: (body temperature, pulse, respiratory rate, heart rate).

3)Anthrometric measurements (body weight, head circumference, body length).

4)Skin examination: (pallor, cyanosis, stained).

5) Umbilical cord examination.

6) Finger nails examination.

B) Investigations including

1. Blood film: Analyzed by sysmex 21-kx cell counter.

2. Kidney function tests: urea and creatinine were done on Bio system A15 chemical auto analyzer by kits supplied by Biosyste (Spain) according to manufacturer instructurer.

3. Lactate measurement: The blood was collected from the vein. The normal value of lactate was considered to be $3.0-4.2 \mathrm{mmol} / \mathrm{L}$.

4. Chest radiography.

\subsection{Statistics}

Descriptive statistics were expressed as range. Distribution of variables was examined using the Kolmogorov-Smirnov test. Quantitative data were analyzed using an independent sample's t test. Qualitative data were analyzed using both the goodness of fit test and test of independence. Pearson regression analysis was used for correlations. Analysis was performed using the SPSS (21). 


\section{Results}

In this table, according to need of resuscitation, the percentage of yes were $30(60 \%)$ and no were 20 (40\%). According to surfactant administration the percentage of yes were $8(16 \%)$ and no were 42 (84\%). Table (1)

According to hospital stay between those infants, range between (4-45) days. Table (2)

According to, the percentage of yes for maternal history of diabetes mellitus (DM) was $10(20 \%)$ and no was $40(80 \%)$. According to maternal history of hypertension, yes in $6(12 \%)$ and no in $44(88 \%)$. Table (3)

According to chest radiography, classic (area of air trapping alternating with areas of atelectasis) in $84 \%$, pnemomediastiunm $8 \%$, pneumothorax in $8 \%$, white out lung in $14 \%$, multiple focal consolidation of chemical pneumonitis in $12 \%$, pulmonary interstitial emphysema in $4 \%$. Table (4)

According to cranial ultrasound between those infants, normal in $22(44 \%)$, mild to moderate hypoxia with early hypoxic-ischemic encephalopathy (HIE) in $16(32 \%)$, moderate to severe hypoxia with moderate HIE in 10 (20\%). Table (5)

According to echocardiography between infants with liquor stained with meconium, normal in 22 (44\%), paten foramen oval (PFO) in $28(56 \%)$, patent ductus arteriosus (PDA) in $8(16 \%)$ interventricular septal hypertrophy (IVSH) in 4 (8\%). Table (6)

According to lactate measurement between male and female, there was no statistically significant difference between both, mean $(4.527,4.40)$ Table (7)

According to the ROC curve analysis, expect need for respiratory support by measurement of lactate fig. (1).

Table (1) Need for resuscitation and surfactant administration between infants with liquor stained with meconium.

\begin{tabular}{llcc}
\hline & & No. & $\%$ \\
\hline Need for & Yes & 30 & 60.0 \\
resuscitation & No & 20 & 40.0 \\
Surfactant & Yes & 8 & 16.0 \\
administration & No & 42 & 84.0 \\
\hline
\end{tabular}

Table (2) Hospital stay between infants with liquor stained with meconium

\begin{tabular}{lcc}
\hline & Range & Mean \pm SD \\
\hline Hospital stay (days) & $4-45$ & $14.24 \pm 11.82$ \\
\hline
\end{tabular}

Table (3) Maternal history between infants with liquor stained with meconium.

\begin{tabular}{llcc}
\hline & & No. & $\%$ \\
\hline Maternal history of & Yes & 10 & 20.0 \\
diabetes mellitus & No & 40 & 80.0 \\
Maternal history of & Yes & 6 & 12.0 \\
hypertension & No & 44 & 88.0 \\
\hline
\end{tabular}

Table (4) Chest $x$ ray between infants with liquor stained with meconium.

\begin{tabular}{llcc}
\hline & & No. & \% \\
\hline & Classic & 42 & 84.0 \\
& Pneumothorax & 4 & 8.0 \\
Pnest x ray & Multiple focal consolidation of Chemical & 4 & 8.0 \\
& pneumonitis & 6 & 12.0 \\
& White out lung & 8 & 16.0 \\
& PIE & 2 & 4.0 \\
\hline
\end{tabular}

Classic: Areas of air trapping alternating with areas of atelectasis

PIE: Pulmonary interstitial emphysema.

Table (5) Cranial ultrasound between infants with liquor stained with meconium.

\begin{tabular}{llcc}
\hline & No. & \% \\
\hline $\begin{array}{l}\text { Cranial } \\
\text { ultrasound }\end{array}$ & $\begin{array}{l}\text { Mild to moderate hypoxia with early } \\
\text { HIE } \\
\text { Moderate to severe hypoxia with } \\
\text { moderate HIE }\end{array}$ & 22 & 44.0 \\
\hline
\end{tabular}

Hypoxic ischemic encephalopathy (HIE) 
Table (6) Echocardiography between infants with liquor stained with meconium.

\begin{tabular}{|c|c|c|c|}
\hline & & No. & $\%$ \\
\hline \multirow{6}{*}{ Echocardiography } & Normal & 22 & 44.0 \\
\hline & PFO & 28 & 56.0 \\
\hline & PDA & 8 & 16.0 \\
\hline & TR & 4 & 8.0 \\
\hline & RVD & 12 & 24.0 \\
\hline & IVSH & 4 & 8.0 \\
\hline
\end{tabular}

Patent Foramen Oval (PFO), Patent Ductus Arteriosus (PDA), Tricuspid Regurgitation (TR), Right ventricle dilatation (RVD), Interventricular septal hypertrophy (IVSH)

Table (7) Comparison between male and female according to lactate measurement.

\begin{tabular}{lccccc}
\hline & & Male & Female & t.test & P. value \\
\hline lactate measurement $(\mathbf{m m o l} / \mathrm{L})$ & Mean \pm SD & $4.527 \pm 1.96$ & $4.40 \pm 1.60$ & .238 & 0.813 \\
\hline
\end{tabular}

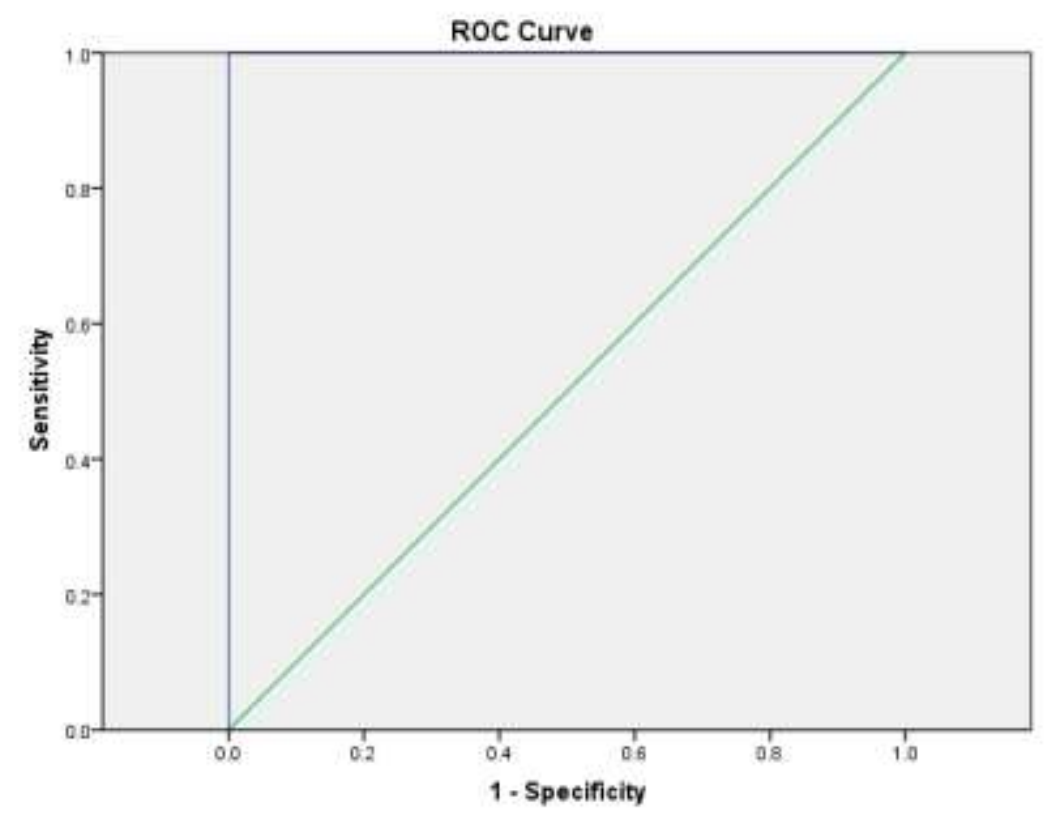

Fig. (1) ROC curve to expect need for respiratory support by measurement of lactate.

\section{Discussion}

We have dealt with 40 children who draw meconium. The proportion of Yes for the need for a revival was $60 \%$ and No $40 \%$.

We agree with Joshi et al., [8] they have shown the necessity to resuscitate children with alcohol stained with meconium up to 53 percent.

We disagree with Dhannaram et al., [9] since only 10.2 percent of babies with liquor stained with meconium required to recover.

Our study has shown that children with liquor stained with meconium who required surfactant treatment were $16 \%$ and $84 \%$ not.

In line with the findings of El shahed et al. [10], they identified a growing demand for the administration of surfactants in children. This occurs because of surfactant therapy which increases respiratory function and index of oxygenation 6 hours after treatment and reduces the breath of meconium.

In Subirà et al. [11], however, no difference has been shown between surfactant washing in babies with meconium breath and restrictions on air leaks or respiratory support time.

In our study, maternal hypertension was developed by $12 \%$, according to the maternal risk factor in the development of meconium intake.

We agree with Vain and Batton [12] that different foetal stress factors, such as maternal hypertension, stimulate the passage of meconium in part from foetal maturation.

We disagree with Rovas et al., [13] examined the risk variables that produce meconium attraction; they showed that the development of meconium attraction was not related to the development of maternal hypertension.

In our study, it was established according to maternal DM (20 percent).

In conjunction with Mohammed et al [14].'s study, the maternal DM was a significant risk factor for liquor stained with meconium 
However, Aviram et al. [15] did not detect a significant relationship between maternal DM and stained meconium fluid.

According to the chest radiograph, $84 \%$ of our work showed air tapping regions alternating with atelectasis.

We agree with Vain \& Batton [12] when the typical chest X-ray was developed in newborns with the breath of meconium, the lung was over-expanded with widely distributed, coarse and patchy infiltrates.

In our study, $12 \%$ of babies suffered from chemical pneumonitis in their chest X-rays.

We disagree with Shaikh et al., [16] when 23.6 percent of patients were found to have pneumonitis.

In this study, $20 \%$ of babies were exposed to all air leaks. Pneumothorax, pneumomediastia and interstitial pulmonary emphysema were determined to be $8 \%, 8 \%$ and $4 \%$.

We agree with Narang et al. [17] that the air leak was found in 15.4 percent of cases.

We disagree with Dargaville \& Copnel, [18] since they found that the total risk of air leak was $9.6 \%$.

Raghu Raman \& Jay Prakash, [19] found that pneumothorax in just $2 \%$ of patients, and pneumothorax in only $2 \%$ of cases.

In this study, we found that, after cranial ultrasonography, $52 \%$ of patients had hypoxia ischemical encephalopathy (HIE), 32\% had mild to moderate $\mathrm{HIE}$, and $20 \%$ had moderate to severe HIE.

We agree with Allanson et al. [20] that 69.7 percent of patients exhibited various degrees of HIE.

We disagree with Raghu Raman \& Jay Prakash, [19], who found that only $4 \%$ of patients had HIE.

20 percent of children with DRM had patent ovals foramen and 16 percent had patent arteriosus ductus.

The Nakwan \& Chitrapatima [20] researchers found patent foramen ovale at $16 \%$ and patent ductus arteriosus at $3 \%$.

In this study, there was no difference in the measurement of lactate between men and women; (4.527, 4.40).

We agree with Zhang \& Ji, [21] they have shown that there are no variations between genders in rest or peak blood lactate levels and lactate removal rates.

According to ROC, rising lactate may be expected to lead to issues like HIE, air leaks and requirement for respiratory assistance in babies with alcohol with meconium stained.

As in the study of yang et al. [22] they analyse serum enzymes in anticipation of the severity of meconium in babies, serum lactate may be a suitable examination of issues such as a need for respiratory assistance in infants with meconium intake.

\section{Conclusion}

Measurement of lactate may predict issues in babies with meconium-treated alcohol such hypoxic ischemic encephalopathy, air leak, lung white and respiratory supports.

\section{References}

[1] A.Greenough. "Respiratory Disorders in the infants". Disorders of the Respiratory Tracts in Children. 8th ed. Philadelphia: Saunders Elsevier.vol.50,pp.358-385,2012.

[2] S.D.Lysander, P.Chandrakala \& M.Jayalalitha. Correlation among $\mathrm{pH}$ in blood and meconium stained deliveries. Int $\mathbf{J}$ Contemp Pediatr.vol.7,pp.60-73,2020.

[3] A.Althaqafi, R.H.Ateeq, D.M.Al-Bukhar. Fetal Outcome and Mode of Delivery in a Patient with Liquor Stained with Meconium. Open Journal of Obstetrics and Gynecology.vol.11,pp.12-19,2021.

[4] M.Rawat. Algorithm to Infants Born Through Liquor Stained with Meconium: J. Perinatal.vol.36,pp.816-823,2017.

[5] G.Van Hall. Lactate kinetics in human tissues at rest and during exercise. Acta Physiol.vol.19,pp.499-508,2010.

[6] A.L.Blomkalns. Lactate - a marker for sepsis and trauma. Emergency Medicine Cardiac Research and Education Group.vol.26,pp.4349,2017.

[7] M.W.Donnino, E.Carney, M.N.Cocchi. Thiamine deficiency in critically ill patients with sepsis. J Crit Care.vol.25,pp.576581,2010 .

[8] S.D.Joshi, G.Jayasakthi, R.Rai. Perinatal outcome of liquor stained with meconium in pregnancy beyond 37 weeks. Int $\mathbf{J}$ Reprod Contracept Obstet Gynecol .vol.10,pp.624-626,2021.

[9] V.Dhannaram, S.Kotapuri \& S.Chitgupikar. Study of early infant outcomes in babies delivered through liquor stained with meconium in a rural teaching hospital. Int $\mathrm{J}$ Contemp Pediatr.vol. 8,pp.26-64,2021.

[10] A.I.El Shahed, P.Dargaville, A.Ohlsson. Surfactant for draw breath of meconium in full term/near term infants. Cochrane Database Syst Rev.vol.3,pp.20-54,2007.

[11] C.Subirà, C.de Haro, R.Magrans. Minimizing Asynchronies in Mechanical Ventilation: Current and Future Trends. Respir Care.vol.63,pp.464-478,2018.

[12] N.E.Vain \& D.G.Batton. Meconium "Draw breath" (or respiratory distress associated with liquor stained with meconium?). Seminars in Fetal and Neonatal Medicine.vol. 22,pp.214-219,2017.

[13] L.Rovas, A.Razbadauskas, E.Boguziene,Risk factors that can lead to development of Draw breath of meconium. Obstet Gynecol Int J.vol.9,pp.208-212,2018.

[14] N.Mohammad, T.Jamal, A.Sohaila. liquor stained with meconium and its neonatal outcome Pakistan journal of medical sciences.vol.34,pp.1392-1396.,2018.

[15]A.Aviram, L.Guy, E.Ashwal. Pregnancy outcome in pregnancies complicated with 
gestational diabetes mellitus and late preterm birth. Diabetes Res Clin Pract.vol.113,pp.198-203,2016.

[16]M.Shaikh, K.A.Irfan Waheed, S.Javoid, Detrimental Complications Of Draw Breath Of Meconium And Their Impact. Journal of Ayub Medical College, Abbottabad.vol.28,pp.506-509,2016.

[17] A.Narang ， P.M.Nair ， O.N.Bhakoo . Management of liquor stained with meconium: a team approach. Indian Pediatrics.vol.30,pp.90-103,1993.

[18] P.A.Dargaville \& B.Copnel, Draw breath of meconium. The Epidemiology of: Incidence, Risk Factors, Therapies, and Outcome; for the Australian and New Zealand Neonatal Network Pediatrics.vol.117,pp.17121721,2006.

[19] T.S.Raghu Raman \& D.G.Jay Prakash, Infant death in meconium stained delivers-A prospective study. Med J Armed Forces India.vol.3,pp.15-18,2017.

[20] J.Q.Zhang \& L.L.Ji, Gender Differences in Peak Blood Lactate Concentration and Lactate Removal. Ann Sports Med Resvol.2,pp.10-88,2016.

[21] M.Y.Yang, I.U.Kim \& H.R.Jeong. Serum Enzymes in Predicting the Severity of Liquor stained with Meconium in Infants. Korean J Perinatal.vol.26,pp.215-221,2015. 